\title{
Montreal Cognitive Assessment
} Functional Test

National Cancer Institute

\section{Source}

National Cancer Institute. Montreal Cognitive Assessment Functional Test. NCI

Thesaurus. Code C123667.

A standardized rating scale developed by Ziad Nasreddine in 1996 to screen for mild cognitive dysfunction and impairment. This instrument assesses the following cognitive domains: attention and concentration, executive functions, memory, language, visuoconstructional skills, conceptual thinking, calculations, and orientation. 